\title{
Dynamic MRI follow-up of radiation encephalopathy in the temporal lobe following nasopharyngeal carcinoma radiotherapy
}

\author{
XIAOFENG ZHOU $^{1}$, XIAOFANG LIAO ${ }^{2}$, XIAOQIU REN $^{1}$, KEWEI XIANG ${ }^{3}$, \\ QIONGGE HU ${ }^{1}$, MINMING ZHANG ${ }^{3}$, HUIJUAN HE ${ }^{2}$, LI SHEN ${ }^{1}$ and QICHUN WEI ${ }^{1}$ \\ ${ }^{1}$ Department of Radiation Oncology, The Second Affiliated Hospital, National Ministry of Education Key Laboratory \\ of Cancer Prevention and Intervention, Zhejiang University School of Medicine, Hangzhou, Zhejiang 310009; \\ ${ }^{2}$ Department of Radiation Oncology, Quzhou Central Hospital, Quzhou, Zhejiang 324000; ${ }^{3}$ Department of Radiology, \\ The Second Affiliated Hospital, Zhejiang University School of Medicine, Hangzhou, Zhejiang 310009, P.R. China
}

Received October 9, 2015; Accepted March 17, 2017

DOI: $10.3892 / \mathrm{ol} .2017 .6199$

\begin{abstract}
The natural course of radiation encephalopathy following nasopharyngeal carcinoma (NPC) radiotherapy remains poorly understood. The present study aimed to investigate the magnetic resonance imaging (MRI) characteristics and evolution of radiation encephalopathy. A series of 162 follow-up MRI examinations from 68 NPC patients with radiation encephalopathy in the temporal lobes were analyzed retrospectively. Each component of radiation encephalopathy was defined as follows: i) contrast enhanced lesions were enhanced lesions on contrast enhanced T1-weighted images (T1WI); ii) white matter lesions were lesions of homogeneous hyperintensity on T2-weighted images (T2WI) and hypointensity on T1WI; iii) cysts were round or oval well-defined lesions of hyperintensity on T2WI; iv) hemosiderin deposition were nodular or annular hypointense lesions with lower hypointense than normal white matter on both T1WI and T2WI; v) gray matter lesions were defined as disruption or erosion of hyperintensity in the cortex on T2WI. Contrast enhanced lesions, white matter lesions, gray matter lesions, cysts and hemosiderin deposition were detected in 105 (100.0\%), 98 (93.3\%), 94 (89.5\%), 2 (1.7\%) and 2 (1.7\%) cases of the 105 initial diagnosed temporal lobe lesions. Contrast enhanced
\end{abstract}

Correspondence to: Dr Kewei Xiang, Department of Radiology, The Second Affiliated Hospital, Zhejiang University School of Medicine, 88 Jiefang Road, Hangzhou, Zhejiang 310009, P.R. China

E-mail: xiangkehz@hotmail.com

Dr Qichun Wei, Department of Radiation Oncology, The Second Affiliated Hospital, National Ministry of Education Key Laboratory of Cancer Prevention and Intervention, Zhejiang University School of Medicine, 88 Jiefang Road, Hangzhou, Zhejiang 310009, P.R. China

E-mail: qichun_wei@zju.edu.cn

Key words: radiation encephalopathy, magnetic resonance imaging, follow-up, nasopharyngeal carcinoma, radiotherapy lesions were the most commonly observed, followed by white matter lesions, gray matter lesions, temporal lobe atrophy, cysts and hemosiderin deposition. In addition, 12 new lesions were identified during the follow-up, 4 of which presented as solid enhanced nodular lesions. Importantly, in 11 of the 117 (9.4\%) affected temporal lobes, solid enhanced nodular lesions were observed to be the only initial abnormalities to occur. For those enhanced nodular lesions measuring $<0.8 \mathrm{~cm}$, no necrosis could be detected. On the contrary, all the contrast enhanced lesions measuring $>2.0 \mathrm{~cm}$ exhibited a necrotic core. To the best of our knowledge, the present study revealed for the first time solid enhanced nodular lesions as the earliest MRI abnormalities of radiation encephalopathy following NPC radiotherapy.

\section{Introduction}

Nasopharyngeal carcinoma (NPC) is a common malignancy in Southeast Asia, particularly in the south of China (1). Radiotherapy is the primary standard radical treatment for NPC. However, radiation affects normal tissues such as the temporal lobe, salivary gland and cochlea, and accounts for various severe delayed complications and sequelae, including radiation encephalopathy, salivary xerostomia and hearing impairment $(2,3)$. Radiation encephalopathy, which was first described in 1930 by Fischer and Holfelder (4), is one of the complications of NPC patients previously treated with radiation. The 3- and 5-year incidence of radiation encephalopathy have been reported to be 10.8 and $34.9 \%$, respectively, following two-dimensional radiotherapy (2D-RT) (5). In Hong Kong, radiation encephalopathy in the temporal lobe accounts for $65 \%$ of all mortalities due to radiation-induced complications in patients with NPC (6). While NPC patients have a long-term survival following chemoradiotherapy, radiation encephalopathy is becoming an important factor affecting NPC patients' quality of life (7). Therefore, it is vital that radiation encephalopathy is correctly identified to avoid unnecessary additional investigations or even inappropriate treatment. Radiation encephalopathy is generally regarded as a progressive and irreversible process (8). Autopsy findings have shown that histological features of radiation encephalopathy include reactive white matter edema, rarefaction of 
myelin or even demyelination, fibrinoid changes in the blood vessels, coagulative necrosis and cysts with reactive gliosis in the walls $(9,10)$. Pathological examination is the gold-standard evaluation. However, brain biopsy is an invasive method, and cannot be used to evaluate the whole lesion or to follow-up the evolution of radiation encephalopathy. Magnetic resonance imaging (MRI) has been demonstrated to be a useful and noninvasive method to evaluate radiation encephalopathy and its evolution $(11,12)$, although the natural course of radiation encephalopathy remains poorly understood.

At present, intensity-modulated radiotherapy (IMRT) is widely employed in the management of NPC, and a highly conformal dose could be delivered to the planned target volume, which enables significantly better organ sparing compared with 2D-RT (5). However, radiation encephalopathy was detected in $\sim 5 \%$ of patients following IMRT (13). Therefore, it is still of clinical interest to analyze the MRI characteristics of radiation encephalopathy.

In the present study, a series of follow-up MRI examinations from 68 NPC patients with radiation encephalopathy in the temporal lobes were analyzed retrospectively. All the patients had been previously treated with radical radiotherapy for NPC. The objective of the present study was to provide an improved understanding of the MRI characteristics and evolution of radiation encephalopathy, which may help to diagnose and treat radiation encephalopathy as early as possible.

\section{Materials and methods}

Clinical data. A total of 68 radiation encephalopathy patients were recruited for the present study. All of them had been previously treated with radical radiotherapy for NPC. The patient cohort consisted of 42 men and 26 women, with a mean age of 50.3 years (range, 22-70 years). Approval for the present retrospective analysis of the patients' data was obtained from the Ethics Committee of the Second Affiliated Hospital, Zhejiang University School of Medicine (Hangzhou, China). Informed consent was obtained when the patients were treated. All patients had pathologically confirmed NPC. Of these, 67 patients received a radical 2D-RT regimen with a dose of $70 \mathrm{~Gy}$, while 1 patient had a first radical 2D-RT course with a dose of $70 \mathrm{~Gy}$, followed by a second radiation course with a dose of 56 Gy for NPC recurrence 13 years after the first radiotherapy.

Regarding the 2D-RT data, the exact radiation dose delivered to the temporal lobes was not available. In order to estimate the approximate volume and dose delivered to the temporal lobes, the dose distribution and dose-volume histogram of a patient treated with 2D-RT were reconstructed (Fig. 1). For this purpose, target volume and organs at risk were contoured by planning computed tomography. The dosimetric parameters V50, V60 and V70, represent the temporal lobe volume that received radiation dose greater than 50,60 and $70 \mathrm{~Gy}$, respectively. The inferior-medial aspect of the temporal lobe received the highest radiation dose, as the V50, V60, V70 and mean dose of the inferior-medial aspect were remarkably higher than those of the whole temporal lobe (Table I). The dose to $0.5 \mathrm{ml}$ of the temporal lobe volume of the bilateral temporal lobes and their inferior-medial aspects were both higher than the dose tolerance of 69 Gy suggested by Sun et al (14).
In the Second Affiliated Hospital, Zhejiang University School of Medicine, NPC patients are recommended to have contrast enhanced nasopharyngeal MRI examination every 3 months in the first 2 years, then every 6 months between 3 and 5 years, and every year thereafter. However, the majority of patients in the present study did not receive regular MRI examination after the third year. Brain MRI was performed if any temporal abnormality was detected. Patients with radiation encephalopathy were identified by head and neck radiologists. The diagnostic standards for radiation encephalopathy of NPC were as follows: i) Having a history of radiotherapy for NPC; ii) having an abnormal signal intensity in the temporal lobe of the brain, which was identified by follow-up MRI examinations; and iii) having temporal lobe lesions, which were detected $\geq 6$ months after radiotherapy (12). Patients with imaging or endoscopic evidence of tumor invading the cavernous sinus or the floor of the middle cranial fossa adjacent to the injured temporal lobes were excluded from the study. Patients with clinical or laboratory evidence of brain infarction, infection or tumors were also excluded from the study.

MRI scan technique. MRI was performed using two 1.5-Tesla systems, Signa HDxt 1.5T (GE Healthcare Life Sciences, Chalfont, UK) and MAGNETOM Sonata 1.5T (Siemens AG, Munich, Germany) at the Second Affiliated Hospital of Zhejiang University School of Medicine. Two MRI protocols were used. The first protocol was a standard follow-up NPC MRI protocol for patients with NPC. Sequences for the first protocol included an axial T2-weighted fat-suppressed sequence, an axial T1-weighted spin-echo sequence and a contrast enhanced T1-weighted spin-echo sequence in the axial, coronal and sagittal planes following a bolus injection of $0.1 \mathrm{mmol}$ gadopentetate dimeglumine (Gd-DTPA) (Magnevist; Schering AG; Bayer Leverkusen, Germany) per $\mathrm{kg}$ of body weight. The second protocol was modified to target the brain. Sequences for the second protocol included an axial T1-weighted spin-echo sequence and an axial T2-weighted turbo spin-echo sequence. Post-contrast axial, sagittal and coronal T1-weighted imaging was performed following an intravenous bolus injection of $0.1 \mathrm{mmol}$ clinical gadolinium chelate per $\mathrm{kg}$ of body weight, and the imaging parameters were the same as for pre-contrast imaging.

MRI appearance of radiation encephalopathy. A total of 68 NPC patients underwent 162 follow-up MRI examinations upon the occurrence of radiation encephalopathy. For each patient, the two temporal lobes were assessed separately. The radiation encephalopathy lesions in the gray and white matter were assessed by MRI, and the diagnosis of each component of radiation encephalopathy was based on the following definitions (6): i) Contrast enhanced lesions were defined as enhanced lesions on contrast enhanced T1-weighted images, with or without necrosis. According to the appearance and the characteristics of lesion enhancement, contrast enhanced lesions were divided into four groups as follows: Solid enhanced nodular lesions; enhanced nodular lesions with a necrotic center; finger-like enhanced lesions; and dotted and patchy enhanced lesions, often along with temporal lobe atrophy. Dotted and patchy enhanced lesions usually have lower enhancement intensity compared with that of the other enhanced lesions 
Table I. Dosimetric parameters of bilateral temporal lobes, and their inferior and medial aspects, in the patient of Fig. 1, who was treated with 70 Gy two-dimensional radiotherapy.

Inferior and medial

Parameter Left temporal lobe Right temporal lobe

aspects of the left temporal lobe

Inferior and medial aspects of the right temporal lobe

\begin{tabular}{|c|c|c|c|c|}
\hline V50 & $16.3 \mathrm{~cm}^{3}$ & $10.5 \mathrm{~cm}^{3}$ & $9.3 \mathrm{~cm}^{3}$ & $6.7 \mathrm{~cm}^{3}$ \\
\hline V60 & $13.9 \mathrm{~cm}^{3}$ & $8.4 \mathrm{~cm}^{3}$ & $8.5 \mathrm{~cm}^{3}$ & $6.1 \mathrm{~cm}^{3}$ \\
\hline V70 & $3.8 \mathrm{~cm}^{3}$ & $1.2 \mathrm{~cm}^{3}$ & $2.2 \mathrm{~cm}^{3}$ & $1.2 \mathrm{~cm}^{3}$ \\
\hline $\mathrm{D}_{0.5 \mathrm{cc}}$ & $72.06 \mathrm{~Gy}$ & $70.85 \mathrm{~Gy}$ & 71.75 Gy & 70.83 Gy \\
\hline $\mathrm{D}_{\text {mean }}$ & $22.34 \mathrm{~Gy}$ & $17.45 \mathrm{~Gy}$ & $62.19 \mathrm{~Gy}$ & $61.30 \mathrm{~Gy}$ \\
\hline
\end{tabular}

V50, temporal lobe volume that received $\geq 50 \mathrm{~Gy}$; V60, temporal lobe volume that received $\geq 60 \mathrm{~Gy}$; V70, temporal lobe volume that received $\geq 70 \mathrm{~Gy} ; \mathrm{D}_{0.5 \mathrm{cc}}$, dose to $0.5 \mathrm{ml}$ of the temporal lobe volume; $\mathrm{D}_{\text {mean }}$, mean dose.
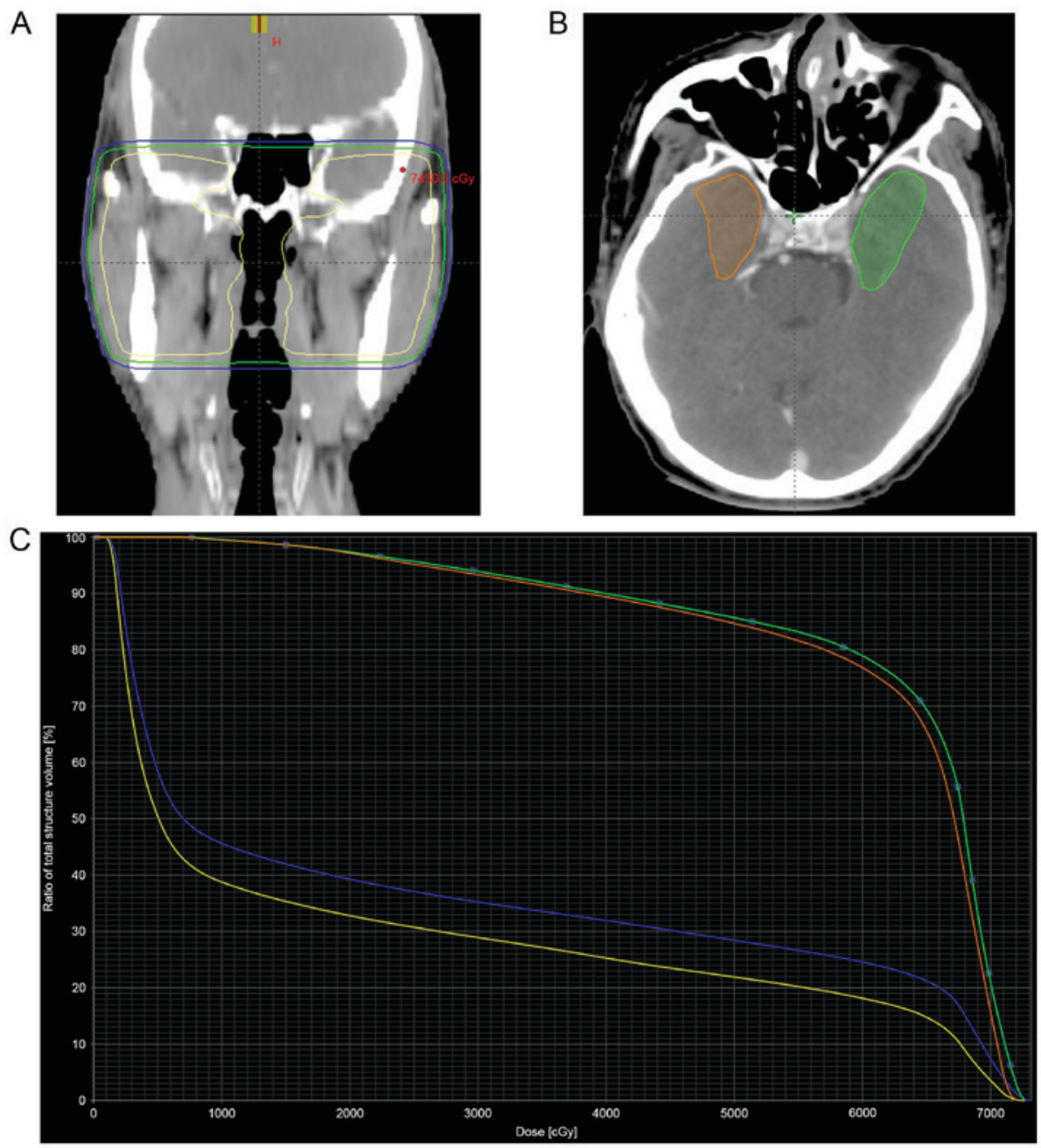

Figure 1. Dose distribution and dose-volume histogram of a patient treated with two-dimensional radiotherapy to 70 Gy. (A) The yellow, green and blue curves represent the isodose curves of 70,60 and $50 \mathrm{~Gy}$, respectively. (B) A transverse planning computed tomography image shows the delineation of the inferior and medial aspects of the bilateral temporal lobes (green, left; orange, right). (C) Dose-volume histogram for the temporal lobes. The blue curve corresponds to the left temporal lobe, while the yellow curve corresponds to the right temporal lobe. The green and orange curves correspond to the inferior and medial aspects of the left and right temporal lobes, respectively.

mentioned above. ii) White matter lesions were defined as lesions of homogeneously high signal intensity on T2-weighted images and low signal intensity on T1-weighted images. White matter lesions were grouped into three categories as follows:
Local (small focal areas in the temporal lobe), moderate (larger confluent areas in the temporal lobe) and diffuse (large diffuse areas extending to the majority of the temporal lobe). iii) Cysts were defined as round or oval well-defined lesions, thin-walled, 
A

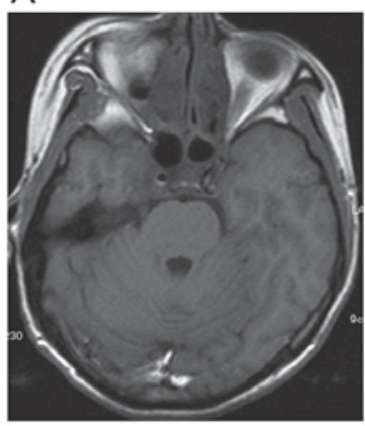

$\mathrm{E}$

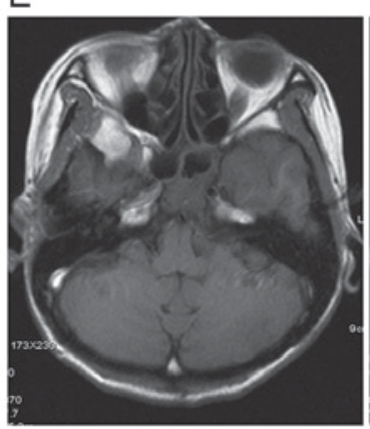

B

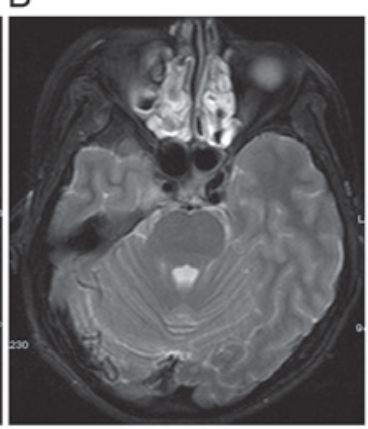

F

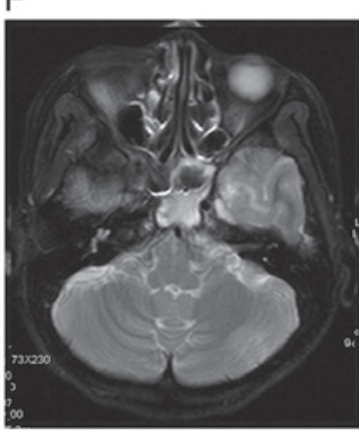

C

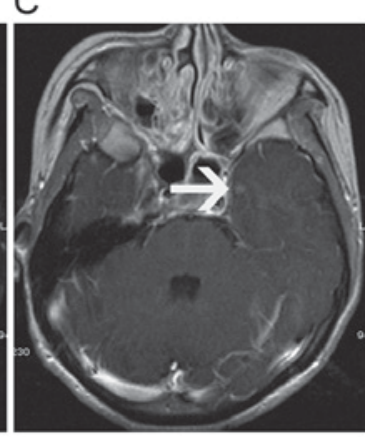

G



D

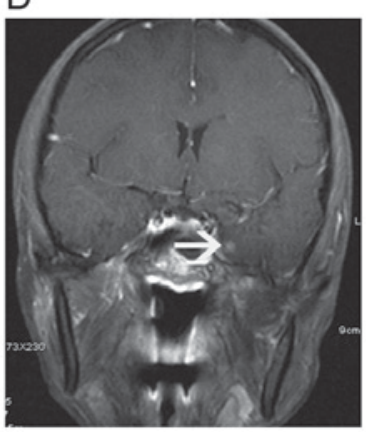

$\mathrm{H}$

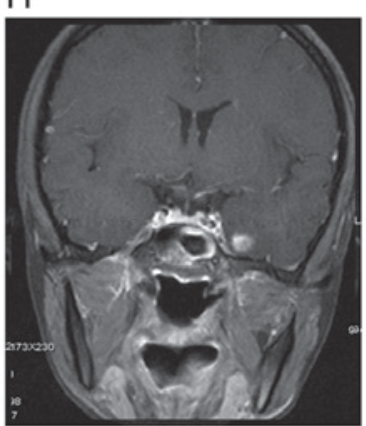

Figure 2. A solid enhanced nodular lesion was detected as the only abnormality to occur at 11 months following radiotherapy. Axial (A) T1- and (B) T2-weighted images show no abnormality in the left temporal lobe, while post-contrast (C) axial and (D) coronal T1-weighted images show a solid enhanced nodular lesion (white arrow) in the inferior and medial aspects of the left temporal lobe. (E-H) Lesion changes in the left temporal lobe are shown in a follow-up MRI examination 5 months later. (E) Axial T1-weighted image shows minimal hypointensity in the left temporal lobe. (F) Axial T2-weighted image shows a local white matter lesion with hyperintensity in the gray-white matter junction of the left temporal lobe. Post-contrast (G) axial and (H) coronal T1-weighted images show an enhanced nodular lesion with necrosis.

of very high signal intensity on T2-weighted images. The cysts were evaluated for size, signal intensity on T1-weighted images and enhancement of the cyst wall following the administration of contrast medium. iv) Nodular or annular hypointense lesions that were more hypointense than normal white matter on both T1- and T2-weighted images were considered as hemosiderin deposition. v) Gray matter lesions of radiation encephalopathy were defined as disruption or erosion of hyperintensity in the cortex on T2-weighted images. vi) Temporal lobe atrophy was considered if there was a subjective decrease in the size of the temporal lobe on MRI. In the process of review of follow-up MRI examinations, the head and neck radiologists excluded patients with clinical or laboratory evidence of brain tumor, infarction, infection or NPC invading the tissue of the middle cranial fossa.

The interval time between radiotherapy and the first detection of any of the above lesions characteristic of radiation encephalopathy was calculated. The median interval time between radiotherapy and the first MRI detection of radiation encephalopathy was 37 months (range, 12-156 months), and the mean interval time was 46.5 months. For the purpose of evaluating the full range and evolution of radiation encephalopathy, the appearance of each component of radiation encephalopathy was assessed in every MRI examination.

Evolution of individual lesions and changes over time. For the purpose of evaluating the evolution of each individual lesion of radiation encephalopathy, each type of lesion was assessed over the course of 162 follow-up MRI examinations upon radiation encephalopathy occurrence. To analyze the overall pattern of abnormalities in radiation encephalopathy, the association between white matter lesions and contrast enhanced lesions was assessed for each MRI examination.

\section{Results}

Initial temporal lobe abnormalities at the first MRI diagnosis of radiation encephalopathy. A total of 105 injured temporal lobes were identified at the initial MRI examination in the 68 cases of radiation encephalopathy analyzed in the present study. Unilateral and bilateral temporal lobe lesions were detected in $31(45.6 \%)$ and $37(54.4 \%)$ of the 68 patients, respectively. For the cases with unilateral lesions, 16 were identified in the left temporal lobe, and the other 15 in the right temporal lobe.

Contrast enhanced lesions were detected in all 105 temporal lobes. Among these, 57 temporal lobes presented with solid enhanced nodular lesions, 24 exhibited enhanced nodular lesions with a necrotic center, 12 had finger-like enhanced lesions, and 12 had dotted and patchy enhanced lesions. White matter lesions were detected in 98 (93.3\%) of the 105 temporal lobes. Local, moderate and diffuse white matter lesions were detected in 41, 21 and 36 temporal lobes, respectively. Gray matter lesions were observed in 94 (89.5\%) of the 105 temporal lobes. Cysts and hemosiderin deposition were noticed in 2 temporal lobes. No temporal lobe atrophy was observed at the MRI examination when radiation encephalopathy was first diagnosed.

It is important to mention that, in 7 affected temporal lobes, solid enhanced nodular lesions were presented as the only initial abnormality (Fig. 2). For these 7 solid enhanced 
A

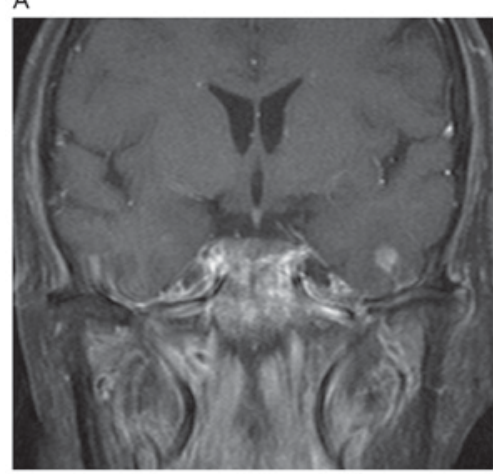

C

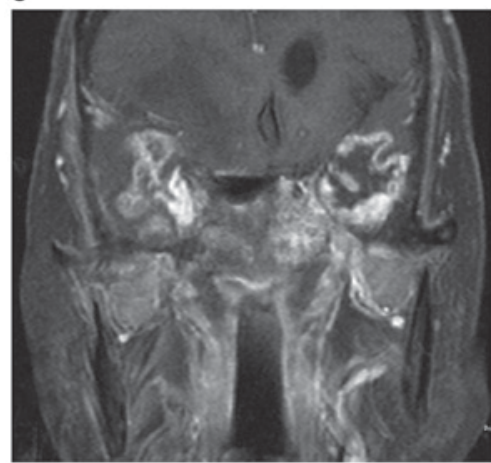

B



D

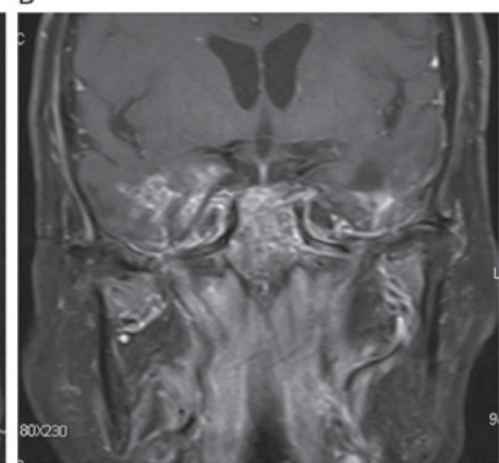

Figure 3. Evolution of a contrast enhanced lesion. (A) A post-contrast coronal T1-weighted image reveals a solid enhanced nodular lesion with homogeneous hyperintensity in the left temporal lobe. (B) The lesion enlarged in size 4 months later, and presented as an enhanced nodular lesion with necrosis. (C) After 6 months, a finger-like enhanced lesion was observed. (D) A dotted and patchy enhanced lesion appeared 12 months later, along with temporal lobe atrophy.

A

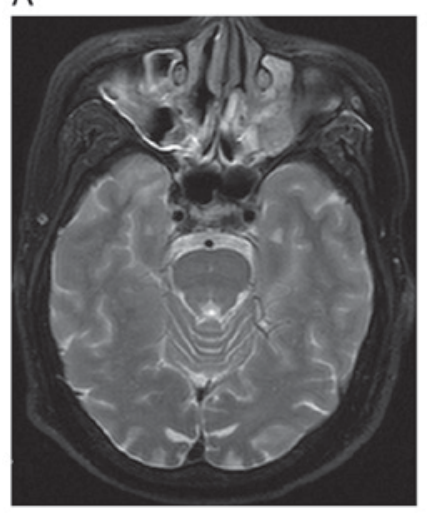

B

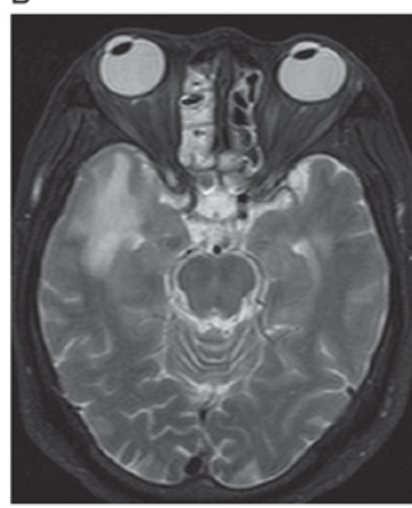

C

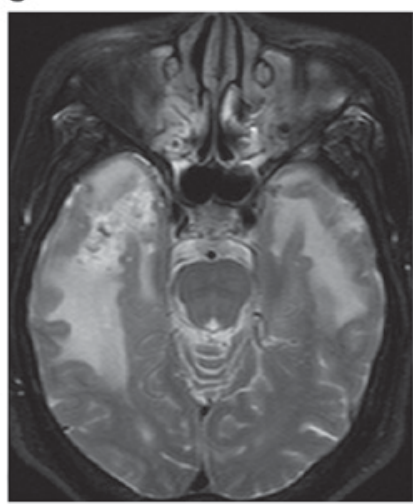

Figure 4. Evolution of a white matter lesion. (A) Axial T2-weighted image reveals a local white matter lesion in the inferior and medial aspects of the right temporal lobe at 29 months following radiotherapy. (B) Axial T2-weighted image shows a larger confluent area of hyperintensity in the right temporal lobe 6 months later, alongside a moderate white matter lesion. (C) Axial T2-weighted image shows a large diffuse region of hyperintensity extending to the majority of the right temporal lobe 5 months later, alongside a diffuse white matter lesion. White matter lesions extended to the gray-white matter junction.

nodular lesions, the previous negative MRI examinations were performed at a median time of 5.5 months prior to MRI detection of radiation encephalopathy. The median time interval was $<10.5$ months (range, 2.5-24.5 months) for patients detected with $\geq 2$ MRI components of radiation encephalopathy, e.g., contrast enhanced lesions, white matter lesions, cysts, gray matter lesions or temporal lobe atrophy.

New MRI abnormalities detected during the follow-up process of radiation encephalopathy. During the follow-up process, 12 cases originally presented as unilateral temporal lobe lesions were observed to exhibit new lesions in the other side of the temporal lobes. Solid enhanced nodular lesions were initially detected as the only abnormality in 4 temporal lobes, and contrast enhanced lesions together with white matter lesions were detected in the other 8 temporal lobes. Regarding the enhanced patterns, solid enhanced nodular lesions, enhanced nodular lesions with a necrotic center and finger-like enhanced lesions were newly detected in 7,1 and 4 temporal lobes, respectively.

New local white matter lesions were noticed in all the 11 temporal lobes where solid enhanced nodular lesions were initially detected as the only abnormality, and the time interval between the detection of a solid enhanced nodular 
lesion and the appearance of local white matter lesions was 3.0-24.5 months (mean, 7.8 months).

Evolution of MRI abnormalities during the follow-up process of radiation encephalopathy. Contrast enhanced lesions usually originated from the gray-white matter junction of the inferior-medial aspect of the temporal lobes. Solid enhanced nodular lesions increased in size and tended to be necrotic at a mean maximal diameter of $1.5 \mathrm{~cm}$ (range, $0.8-3.0 \mathrm{~cm}$ ). The incidence rate of a necrotic core for enhanced nodular lesions of size $<1,1.0-1.5,1.6-2.0$ and $>2 \mathrm{~cm}$ in diameter was 7.7, 41.5, 81.8 and $100.0 \%$, respectively. For those enhanced nodular lesions measuring $<0.8 \mathrm{~cm}$, no necrosis could be detected. On the contrary, all the contrast enhanced lesions that were larger than $2 \mathrm{~cm}$ displayed a necrotic core. Enhanced nodular lesions with a necrotic center continued to increase in size, extended to the gray-white matter junction of the superior-lateral aspect of the temporal lobes and formed finger-like enhanced lesions. Subsequently, the lesions decreased and regressed both in size and signal intensity, as indicated by the dotted and patchy enhanced lesions shown in Fig. 3.

White matter lesions also originated from the gray-white matter junction of the inferior-medial aspect of the temporal lobes, and developed from local lesions to moderate and diffuse white matter lesions with time (Fig. 4). Diffuse white matter lesions decreased both in size and signal intensity with the occurrence of temporal lobe atrophy. However, the development from diffuse white matter lesion to local white matter lesion was not detected in the present study.

In the current study, 77 temporal lobe lesions underwent $\geq 2$ follow-up MRI examinations following radiation encephalopathy occurrence. The evolution pattern of contrast enhanced lesions and white matter lesions could be classified as increasing, decreasing or stable in extent or size. Contrast enhanced lesions and white matter lesions were observed to change with the same evolution pattern in 72 (93.5\%) of 77 temporal lobes: Increasing, decreasing, increasing followed by decreasing and stable in $33(42.9 \%), 14(18.2 \%), 13(16.9 \%)$ and $12(15.6 \%)$ of the 77 temporal lobes, respectively (Fig. 5). The median interval time between the first MRI examination of radiation encephalopathy and lesion increasing was 10.5 months, while the corresponding interval time for decreasing lesions was 20.5 months. In the other $5(6.5 \%)$ temporal lobes, different evolution patterns were observed: White matter lesion stable and contrast enhanced lesion increasing in 3 temporal lobes, and white matter lesion decreasing and contrast enhanced lesion stable in 2 temporal lobes (Table II).

All the cysts that occurred subsequent to necrosis presented as round or oval well-defined lesions with hypointensity on T1-weighted images and hyperintensity on T2-weighted images. Cyst wall enhancement could be occasionally detected on post-contrast T1-weighted images (Fig. 6). Hemosiderin deposition was observed as hypointensity on both T1- and T2-weighted images, accompanied by hemorrhage of hyperintensity on both T1- and T2-weighted images in certain cases.

Gray matter lesions arose in $89.5 \%$ of temporal lobes at the first MRI detection of radiation encephalopathy, and reached 98.3\% at the last follow-up. Gray matter lesions progressed mildly and slowly, and presented as disruption or erosion of the cortex on both T1- and T2-weighted images, with contrast
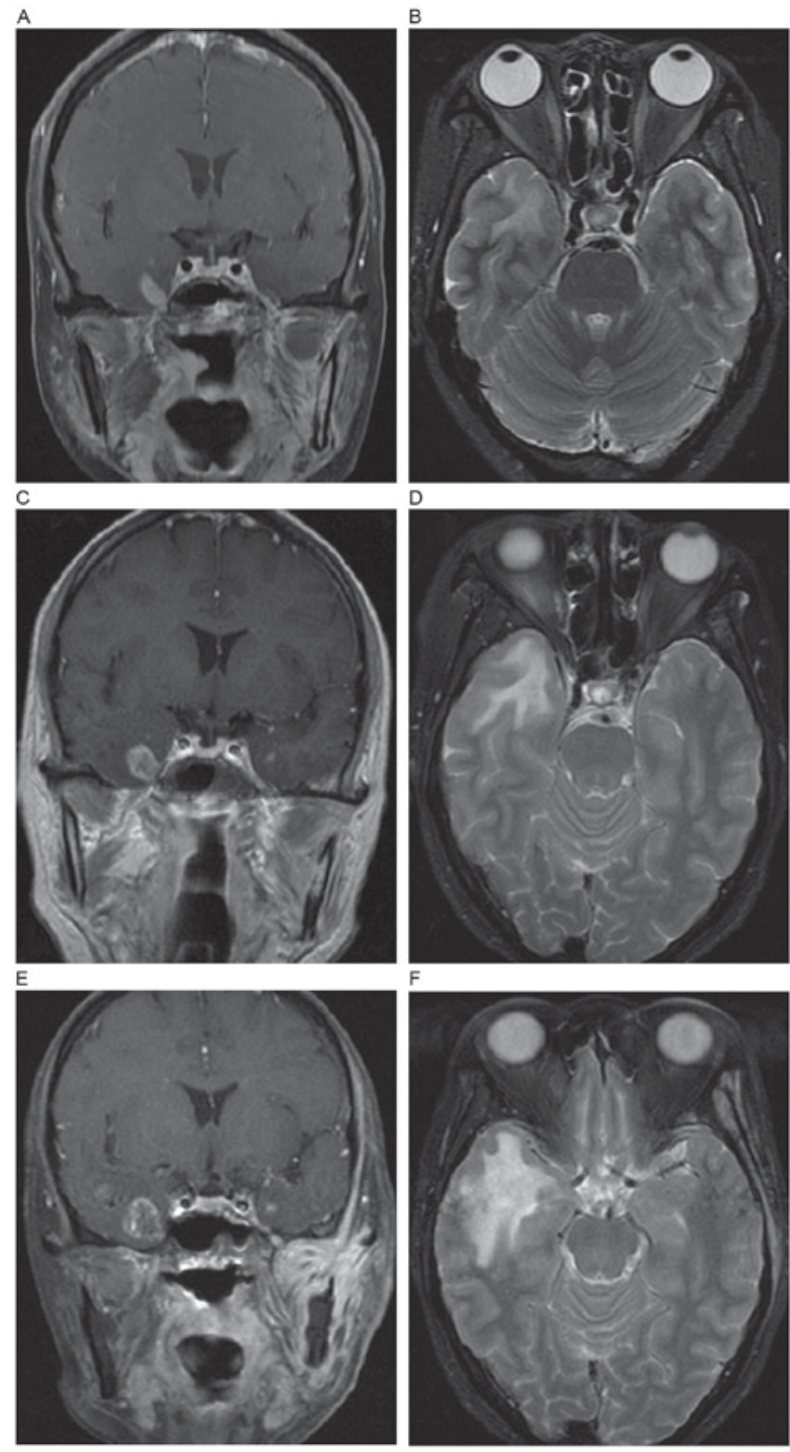

Figure 5. Evolution pattern of an increasing white matter lesion and an increasing contrast enhanced lesion. (A) Post-contrast coronal T1-weighted image shows a solid enhanced nodular lesion in the right temporal lobe. (B) Axial T2-weighted image shows a local white matter lesion in the right temporal lobe. (C-F) In the following two magnetic resonance imaging examinations, the contrast enhanced lesion extended successively, accompanied by extending of the white matter lesion. (C) The contrast enhanced lesion in the right temporal lobe extended to form an enhanced nodular lesion with a necrotic center. (D) The local white matter lesion in the right temporal lobe extended to form a moderate white matter lesion. (E) The enhanced nodular lesion with a necrotic center in the right temporal lobe continued to extend to be a larger size. (F) The moderate white matter lesion in the right temporal lobe extended to form a diffuse white matter lesion.

enhancement on post-contrast T1-weighted images (Fig. 7). Temporal lobe atrophy occurred at the late stage of radiation encephalopathy.

The MRI abnormalities detected during the continuous follow-up MRI examinations are presented in Table III, which indicated the dynamic changes of the different abnormal MRI components.

At the end of the follow-up period, unilateral radiation encephalopathy was noticed in 19 patients, while bilateral radiation encephalopathy was detected in 49 patients, with a total of 117 injured temporal lobes in all the 68 cases. Contrast enhanced lesions and white matter lesions were detected 
Table II. Association between WMLs and CELs in temporal lobes, and their changes over time.

\begin{tabular}{|c|c|c|c|c|}
\hline Lesion evolution & $\begin{array}{l}\text { CEL increasing, } \\
\mathrm{n}(\%)\end{array}$ & $\begin{array}{c}\text { CEL decreasing, } \\
\mathrm{n}(\%)\end{array}$ & $\begin{array}{l}\text { CEL increasing followed } \\
\text { by decreasing, n }(\%)\end{array}$ & $\begin{array}{l}\text { CEL stable, } \\
\text { n }(\%)\end{array}$ \\
\hline WML increasing & $33(42.9)^{\mathrm{a}}$ & $0(0.0)$ & $0(0.0)$ & $0(0.0)$ \\
\hline WML decreasing & $0(0.0)$ & $14(18.2)$ & $0(0.0)$ & $2(2.6)$ \\
\hline WML increasing followed by decreasing & $0(0.0)$ & $0(0.0)$ & $13(16.9)$ & $0(0.0)$ \\
\hline WML stable & $3(3.9)$ & $0(0.0)$ & $0(0.0)$ & $12(15.6)$ \\
\hline
\end{tabular}

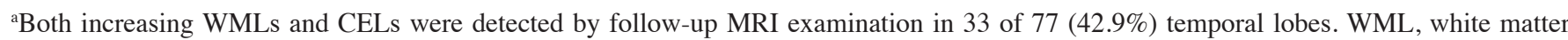
lesion; CEL, contrast enhanced lesion.
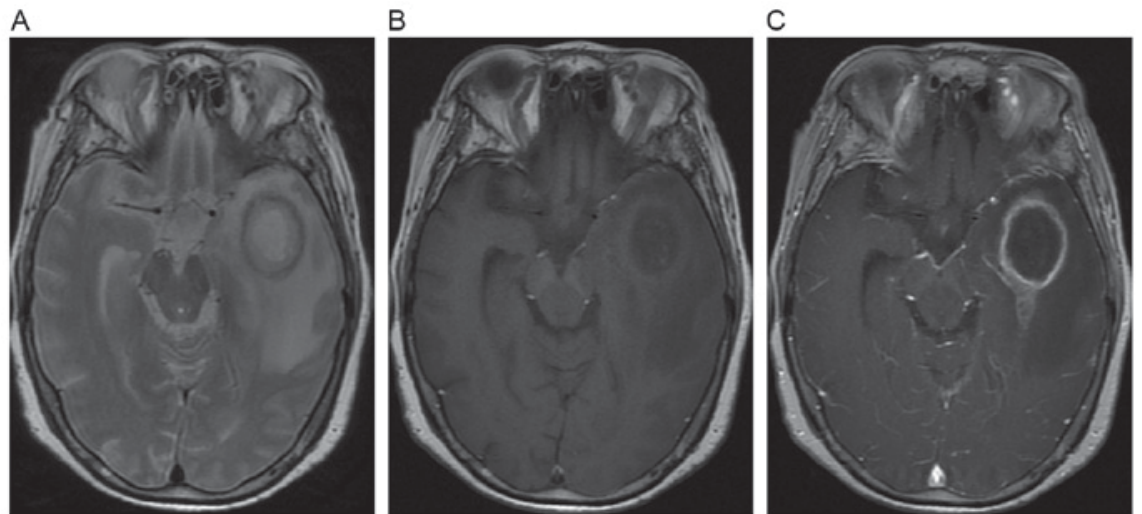

Figure 6. Example of magnetic resonance images of a cyst lesion from a 55-year-old female patient. (A) Axial T2-weighted image shows an oval cyst with inner hyperintensity and outer annular hypointensity. (B) Axial T1-weighted image shows the oval cyst with inner hypointensity and outer annular hyperintensity. (C) Axial post-contrast T1-weighted image shows the cyst with annular enhancement.

in all the 117 temporal lobes. At the late stage of radiation encephalopathy, the majority of diffuse white matter lesions were detected together with finger-like or dotted and patchy enhanced lesions. Gray matter lesions, temporal lobe atrophy, cysts and hemosiderin deposition were detected in 115 (98.3\%), $24(20.5 \%)$ and $6(5.1 \%)$ of the 117 temporal lobes, respectively.

\section{Discussion}

To the best of our knowledge, the present study is the first to describe solid enhanced nodular lesions as the earliest MRI abnormalities of radiation encephalopathy following NPC radiotherapy. In the present study, at the time of the initial MRI diagnosis of radiation encephalopathy, contrast enhanced lesions were detected in all the injured temporal lobes, while white matter lesions were detected in $93.3 \%$ of cases, followed by gray matter lesions, cysts and hemosiderin deposition. Importantly, a solid enhanced nodular lesion was observed to be the first and only MRI abnormality of radiation encephalopathy in 11 out of the 117 involved temporal lobes, and was the only imaging pattern of MRI abnormality for several months (range, 3.0-24.5 months; mean, 7.8 months) before a second component of MRI abnormality (usually a local white matter lesion) appeared in the MRI follow-up. Concerning the earliest MRI abnormality, the present findings are inconsistent with previous studies, in which contrast enhanced lesions were reported to occur subsequently or concurrently with white matter lesions. In the study by Wang et al (15), white matter
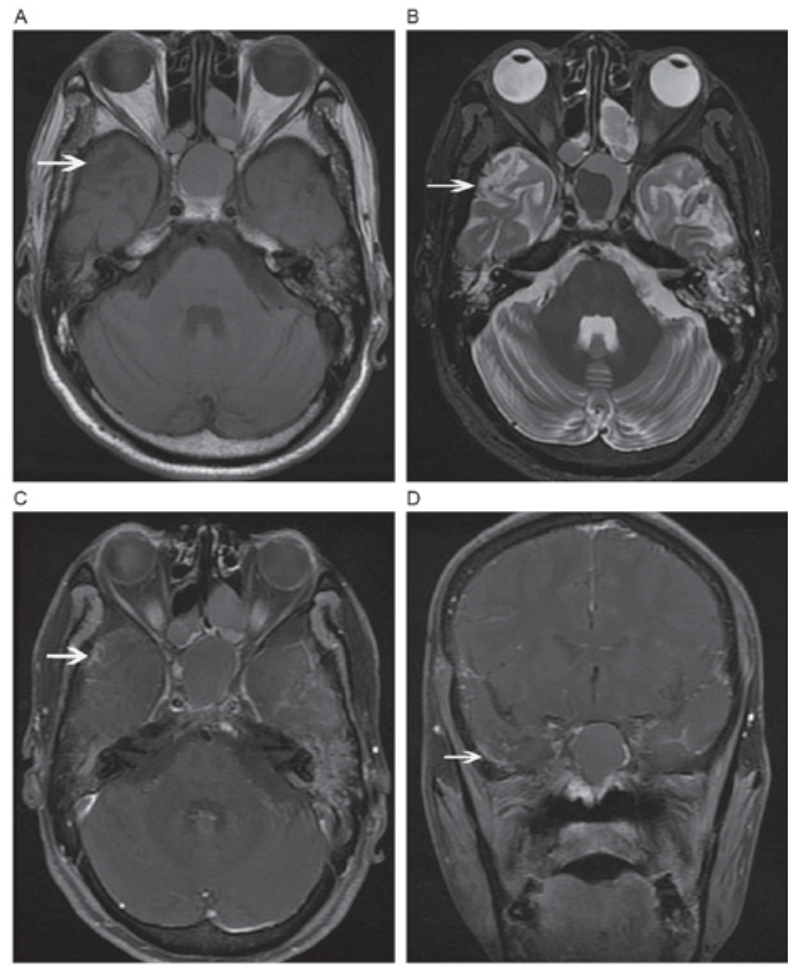

Figure 7. Magnetic resonance images of a gray matter lesion. Axial (A) T1and (B) T2-weighted images show disruption of the gray matter (white arrow) together with temporal lobe atrophy. Post-contrast (C) axial and (D) coronal T1-weighted images show dotted contrast enhancement of gray matter in the cortex (white arrow). Panels B and D show temporal lobe atrophy. 
Table III. Composition of abnormal MRI components at continuous FU MRI examinations.

\begin{tabular}{|c|c|c|c|c|c|c|c|c|c|c|}
\hline \multirow[b]{2}{*}{ Radiation encephalopathy components } & \multicolumn{2}{|c|}{$\begin{array}{c}\text { Diagnostic } \\
\text { MRI }^{\mathrm{a}} \\
(\mathrm{n}=117)\end{array}$} & \multicolumn{2}{|c|}{$\begin{array}{c}\text { 1st MRI } \\
\text { FU (n=77) }\end{array}$} & \multicolumn{2}{|c|}{$\begin{array}{l}\text { 2nd MRI } \\
\text { FU }(n=51)\end{array}$} & \multicolumn{2}{|c|}{$\begin{array}{c}\text { 3rd MRI } \\
\text { FU }(n=20)\end{array}$} & \multicolumn{2}{|c|}{$\begin{array}{l}\text { 4th MRI } \\
\text { FU }(n=5)\end{array}$} \\
\hline & $\mathrm{N}$ & $\%$ & $\mathrm{~N}$ & $\%$ & $\mathrm{~N}$ & $\%$ & $\mathrm{~N}$ & $\%$ & $\mathrm{~N}$ & $\%$ \\
\hline Contrast enhanced lesions & 117 & 100.0 & 77 & 100.0 & 51 & 100.0 & 20 & 100.0 & 5 & 100.0 \\
\hline Solid enhanced nodular lesions & 64 & 54.7 & 20 & 26.0 & 2 & 3.9 & 0 & 0.0 & 1 & 20.0 \\
\hline Enhanced nodular lesions with necrotic centers & 25 & 21.4 & 25 & 32.5 & 12 & 23.5 & 4 & 20.0 & 1 & 20.0 \\
\hline Finger-like enhanced lesions & 16 & 13.7 & 21 & 27.3 & 19 & 37.3 & 6 & 30.0 & 0 & 0.0 \\
\hline Dotted and patchy enhanced lesions & 12 & 10.3 & 11 & 14.3 & 18 & 35.3 & 10 & 50.0 & 3 & 60.0 \\
\hline White matter lesions & 106 & 90.6 & 77 & 100.0 & 51 & 100.0 & 20 & 100.0 & 5 & 100.0 \\
\hline Local & 44 & 37.6 & 17 & 22.1 & 2 & 3.9 & 0 & 0.0 & 0 & 0.0 \\
\hline Moderate & 21 & 17.9 & 20 & 26.0 & 9 & 17.6 & 3 & 15.0 & 3 & 60.0 \\
\hline Diffuse & 41 & 35.0 & 40 & 51.9 & 40 & 78.4 & 17 & 85.0 & 2 & 40.0 \\
\hline Cysts & 2 & 1.7 & 0 & 0.0 & 4 & 7.8 & 2 & 10.0 & 2 & 40.0 \\
\hline Hemosiderin deposition & 2 & 1.7 & 3 & 3.9 & 3 & 5.9 & 3 & 15.0 & 1 & 20.0 \\
\hline Gray matter lesions & 98 & 83.8 & 75 & 97.4 & 51 & 100.0 & 20 & 100.0 & 5 & 100.0 \\
\hline Temporal lobe atrophy & 0 & 0.0 & 11 & 14.3 & 8 & 15.7 & 9 & 45.0 & 4 & 80.0 \\
\hline
\end{tabular}


imaging; FU, follow-up.

lesions were reported to be the only lesion to occur alone, and were detected in $100 \%$ of all the injured temporal lobes. On the contrary, only $82.5 \%$ of the injured temporal lobes exhibited contrast enhanced lesions in that study. In another report, Chan et al (6) also reported white matter lesions as the only abnormal finding of MRI, and contrast enhanced lesions were observed in $89 \%$ of all the injured temporal lobes. The median time interval between previous negative MRI examinations and a subsequent MRI detections of radiation encephalopathy was 10.5 months in the present study, compared with 20.5 months in the study by Wang et al (15). Shorter MRI examination intervals may have the advantage of identifying earlier MRI abnormality of radiation encephalopathy.

Although NPC patients are recommended to receive contrast enhanced nasopharyngeal MRI examination according to the aforementioned follow-up schedule, the majority of patients in the present study did not receive regular MRI examination after the third year. As patients with radiation encephalopathy are usually asymptomatic at the early stage, the injured temporal lobes were detected incidentally in numerous patients. Thus, the first MRI detection of radiation encephalopathy could occur at any stage of the disease course, from early to late stage. Therefore, there is an obvious limitation to estimate the exact timing of the occurrence and development of each MRI pattern of radiation encephalopathy. Theoretically, shorter intervals between each MRI examination may reveal more information closer to the natural history of radiation encephalopathy. The median time interval between a previous negative MRI examination and a subsequent MRI detection of radiation encephalopathy was 10.5 months in the present series, compared with 20.5 months in the study by Wang et al (15). At the first MRI diagnosis of radiation encephalopathy, solid enhanced nodular lesions were detected as the only initial abnormality in 7 affected temporal lobes. For these 7 cases with solid enhanced nodular lesions, the time interval between the first MRI detection of radiation encephalopathy and the previous negative MRI examination was shorter than that for those with multiple MRI components at the time of detection of radiation encephalopathy (5.5 vs. 10.5 months, respectively). In the follow-up process, 12 cases that originally presented as unilateral temporal lobe lesions were observed to exhibit new lesions in the other side of the temporal lobes. Solid enhanced nodular lesions were initially detected as the first and only lesion to occur in 4 of these 12 temporal lobes. The time from the previous negative MRI scan was 6.8 months in these 4 cases, which was shorter than that of the other 8 cases with multiple MRI components. Therefore, it can be proposed that solid enhanced nodular lesions may be the earliest MRI abnormalities to occur alone in the majority of radiation encephalopathy cases.

In the present study, contrast enhanced lesions were detected in all the injured temporal lobes, and were the most common component of MRI abnormalities in radiation encephalopathy. Of all the contrast enhanced lesions, $>50 \%$ were solid enhanced nodules without necrosis on MRI, which were located in the inferior-medial aspects of the temporal lobe, where the highest irradiation dose was delivered. Solid enhanced nodular lesions were usually small, and as the lesions increased in size, the lesions tended to be necrotic; thus, they were termed enhanced nodular lesions with necrotic centers. Taken solid enhanced nodular lesions and enhanced nodular lesions with necrotic centers together, only $7.7 \%$ of the lesions measuring $<1 \mathrm{~cm}$ displayed a necrotic center; $93.1 \%$ of the lesions measuring $>1.5 \mathrm{~cm}$ exhibited necrotic centers; and all the lesions measuring $>2 \mathrm{~cm}$ exhibited necrotic centers. These findings are in concordance with 
the study by Wang et al (15), who reported that all the lesions with size $\geq 2 \mathrm{~cm}$ exhibited a central necrotic core. Enhanced nodular lesions with a necrotic center continued to enlarge and extended to the gray-white matter junction of the superior-lateral aspect of the temporal lobes, forming finger-like enhanced lesions. Subsequently, the lesions decreased and regressed both in size and signal intensity, and formed dotted and patchy enhanced lesions.

In the present study, white matter lesions were the second most common MRI appearance at the first MRI detection of radiation encephalopathy, and were observed in $93.3 \%$ of all the injured temporal lobes. Local or moderate white matter lesions were detected in the majority of lobes, while diffuse lesions were noticed in 1/3 of cases. Diffuse white matter lesions were always detected together with finger-like or dotted and patchy enhanced lesions. In addition, local white matter lesions were detected predominantly in the inferior-medial aspects of the temporal lobe, where the highest irradiation dose was delivered. During the follow-up process, these white matter lesions extended to the gray-white matter junction of the superior-lateral aspect of the temporal lobes. At the end of the follow-up period, white matter lesions were detected in all the injured temporal lobes, with $61.5 \%$ of them being diffuse white matter lesions.

Gray matter lesions arose in $\sim 80.0 \%$ of all temporal lobes at the first MRI detection of radiation encephalopathy, and reached $98.3 \%$ at the last follow-up. Chan et al (6) reported a similar incidence of $87.7 \%$ (50 of 57 injured temporal lobes) in a previous study. Gray matter lesions were often presented as disruption or erosion of the cortex in the focal region, and progressed mildly and slowly. Gray matter may be more tolerant to radiation than white matter due to a different microstructure $(16,17)$. Cysts and hemosiderin deposition often occurred following necrosis, which is consistent with previous studies $(6,13,14,18)$.

The median interval time between radiotherapy and the first MRI detection of radiation encephalopathy was 37 months in our series, which is similar to the 36 months reported by Wang et al (15). However, longer periods (i.e., 55.9 and 44.5 months) were reported by Norris et al (18) and Mao et al (13), respectively. In the present study, bilateral temporal lobes lesions were detected in $54.4 \%$ of patients when a diagnosis of radiation encephalopathy was established, and this number increased to $72.1 \%$ at the last follow-up. The incidence of bilateral radiation encephalopathy has been reported to be $67.6 \%$ (6), $54.8 \%$ (13) and $77.8 \%$ (15). Radiation encephalopathy in bilateral temporal lobes appears to be more frequently observed with 2D-RT compared with IMRT (5). This is possibly due to the fact that parallel opposed beams were administered to the NPC radiation field, and both temporal lobes received approximately the same radiation dose, as demonstrated in Fig. 1. In the era of IMRT, better organ sparing compared with 2D-RT is possible to achieve clinically. As a result, if the tumor is close to one side of the temporal lobe, the other side will not receive an unnecessary high dose of radiation (14). A low bilateral radiation encephalopathy rate of $27.8 \%$ has been reported for IMRT (13), and it could be speculated that radiation encephalopathy in unilateral other than bilateral temporal lobes will be more common in the following years.
In conclusion, the present study is the first to describe solid enhanced nodular lesions as the earliest MRI abnormalities of radiation encephalopathy following NPC radiotherapy. Contrast enhanced lesions and white matter lesions arose from the inferior-medial aspects of temporal lobes, where the highest irradiation dose was delivered, and extended to the gray-white matter junction of the superior-lateral aspect of the temporal lobes. Solid enhanced nodular lesions appeared at the early stage of radiation encephalopathy, followed by enhanced nodular lesions with a necrotic center and finger-like enhanced lesions. As the lesions increased in size, dotted and patchy enhanced lesions were detected at the late stage of radiation encephalopathy. Local white matter lesions developed into moderate lesions, and then diffuse white matter lesions with time. In the majority of cases, contrast enhanced lesions and white matter lesions were observed to change with the same evolution pattern. Temporal lobe atrophy, cysts and hemosiderin deposition occurred at the late stage of radiation encephalopathy. To the best of our knowledge, the present study revealed, for the first time, solid enhanced nodular lesions as the earliest MRI abnormalities of radiation encephalopathy following NPC radiotherapy. These results may help to the early diagnosis of radiation encephalopathy.

\section{Acknowledgements}

The present study was supported by the National Natural Science Foundation of China (grant nos. 81071823 and 81201811) and Zhejiang University Research Foundation (grant no. 11-491020-311).

\section{References}

1. Cao SM, Simons MJ and Qian CN: The prevalence and prevention of nasopharyngeal carcinoma in China. Chin J Cancer 30: 114-119, 2011

2. Lee AW, Ng WT, Hung WM, Choi CW, Tung R, Ling YH, Cheng PT, Yau TK, Chang AT, Leung SK, et al: Major late toxicities after conformal radiotherapy for nasopharyngeal carcinoma-patient-and treatment-related risk factors. Int J Radiat Oncol Biol Phys 73: 1121-1128, 2009.

3. Jen YM, Shih R, Lin YS, Su WF, Ku CH, Chang CS, Shueng PW, Hwang JM, Liu DW, Chao HL, et al: Parotid gland-sparing 3-dimensional conformal radiotherapy results in less severe dry mouth in nasopharyngeal cancer patients: A dosimetric and clinical comparison with conventional radiotherapy. Radiother Oncol 75: 204-209, 2005.

4. Fischer AW and Holfelder H: Lokales amyloid in gehirn. Dtsch Zchir 227: 475-483, 1930.

5. Zhou GQ, Yu XL, Chen M, Guo R, Lei Y, Sun Y, Mao YP, Liu LZ, Li L, Lin AH and Ma J: Radiation-induced temporal lobe injury for nasopharyngeal carcinoma: A comparison of intensity-modulated radiotherapy and conventional two-dimensional radiotherapy. PLoS One 8: e67488, 2013.

6. Chan YL, Leung SF, King AD, Choi PH and Metreweli C: Late radiation injury to the temporal lobes: Morphorlogic evaluation at MR imaging. Radiology 213: 800-807, 1999.

7. Lee AW, Ng SH, Ho JH, Tse VK, Poon YF, Tse CC, Au GK, O SK, Lau WH and Foo WW: Clinical diagnosis of late temporal lobe necrosis following radiation therapy for nasopharyngeal carcinoma. Cancer 61: 1535-1542, 1988.

8. Valk PE and Dillon WP: Radiation injury of the brain. AJNR Am J Neuroradiol 12: 45-62, 1991.

9. Husain MM and Garcia JH: Cerebral 'radiation necrosis': Vascular and glial features. Acta Neuropathol 36: 381-385, 1976.

10. Okeda R and Shibata T: Radiation encephalopathy-an autopsy case and some comments on the pathogenesis of delayed radionecrosis of central nervous system. Acta Pathol Jpn 23: 867-883, 1973. 
11. Chan KC, Khong PL, Cheung MM, Wang S, Cai KX and Wu EX: MRI of late microstructural and metabolic alterations in radiation-induced brain injuries. J Magn Reson Imaging 29: 1013-1020, 2009.

12. Greene-Schloesser D, Robbins ME, Peiffer AM, Shaw EG, Wheeler KT and Chan MD: Radiation-induced brain injury: A review. Front Oncol 2: 73, 2012.

13. Mao YP,Zhou GQ,Liu LZ, Guo R, Sun Y,Li L,Lin AH,Zeng MS, Kang TB, Jia WH, et al: Comparison of radiological and clinical features of temporal lobe necrosis in nasopharyngeal carcinoma patients treated with $2 \mathrm{D}$ radiotherapy or intensity-modulated radiotherapy. Br J Cancer 110: 2633-2639, 2014.

14. Sun Y, Zhou GQ, Qi ZY, Zhang L, Huang SM, Liu LZ, Li L, Lin AH and Ma J: Radiation-induced temporal lobe injury after intensity modulated radiotherapy in nasopharyngeal carcinoma patients: A dose-volume-outcome analysis. BMC Cancer 13: 397, 2013.
15. Wang YX, King AD, Zhou H, Leung SF, Abrigo J, Chan YL, Hu CW, Yeung DK and Ahuja AT: Evolution of radiation-induced brain injury: MR imaging-based study. Radiology 254: 210-218, 2010.

16. Medin PM, Foster RD, van der Kogel AJ, Sayre JW, McBride WH and Solberg TD: Spinal cord tolerance to single-fraction partial-volume irradiation: A swine model. Int J Radiat Oncol Biol Phys 79: 226-232, 2011.

17. Medin PM, Foster RD, van der Kogel AJ, Sayre JW, McBride WH and Solberg TD: Spinal cord tolerance to reirradiation with single-fraction radiosurgery: A swine model. Int J Radiat Oncol Biol Phys 83: 1031-1037, 2012.

18. Norris AM, Carrington BM and Slevin NJ: Late radiation change in the CNS: MR imaging following gadolinium enhancement. Clin Radiol 52: 356-362, 1997. 